\title{
Alien limb in the corticobasal syndrome: phenomenological characteristics and relationship to apraxia
}

\author{
David J. Lewis-Smith ${ }^{1,2,3} \cdot$ Noham Wolpe $e^{1,4}$ (B) Boyd C. P. Ghosh ${ }^{1,5} \cdot$ James B. Rowe ${ }^{1,4,6}$
}

Received: 13 September 2019 / Revised: 8 December 2019 / Accepted: 9 December 2019 / Published online: 9 January 2020

(c) The Author(s) 2020

\begin{abstract}
Alien limb refers to movements that seem purposeful but are independent of patients' reported intentions. Alien limb often co-occurs with apraxia in the corticobasal syndrome, and anatomical and phenomenological comparisons have led to the suggestion that alien limb and apraxia may be causally related as failures of goal-directed movements. Here, we characterised the nature of alien limb symptoms in patients with the corticobasal syndrome $(n=30)$ and their relationship to limb apraxia. Twenty-five patients with progressive supranuclear palsy Richardson syndrome served as a disease control group. Structured examinations of praxis, motor function, cognition and alien limb were undertaken in patients attending a regional specialist clinic. Twenty-eight patients with corticobasal syndrome (93\%) demonstrated significant apraxia and this was often asymmetrical, with the left hand preferentially affected in 23/30 (77\%) patients. Moreover, 25/30 (83\%) patients reported one or more symptoms consistent with alien limb. The range of these phenomena was broad, including changes in the sense of ownership and control as well as unwanted movements. Regression analyses showed no significant association between the severity of limb apraxia and either the occurrence of an alien limb or the number of alien limb phenomena reported. Bayesian estimation showed a low probability for a positive association between alien limb and apraxia, suggesting that alien limb phenomena are not likely to be related to severity apraxia. Our results shed light on the phenomenology of these disabling and as yet untreatable clinical features, with relevance to theoretical models of voluntary action.
\end{abstract}

Keywords Alien limb syndrome $\cdot$ Anarchic hand syndrome $\cdot$ Apraxia $\cdot$ Corticobasal syndrome $\cdot$ Volition $\cdot$ Sense of ownership · Sense of agency

David J. Lewis-Smith and Noham Wolpe contributed equally to this work.

Noham Wolpe

nw305@medschl.cam.ac.uk

1 Department of Clinical Neurosciences, University of Cambridge, Herchel Smith Building, Cambridge CB2 0SZ, UK

2 Translational and Clinical Research Institute, Newcastle University, Newcastle upon Tyne NE2 4HH, UK

3 Department of Clinical Neurosciences, Newcastle upon Tyne Hospitals NHS Foundation Trust, Newcastle upon Tyne NE1 4LP, UK

4 MRC Cognition and Brain Sciences Unit, Cambridge CB2 7EF, UK

5 Wessex Neuroscience Centre, Southampton SO16 6YD, UK

6 Cambridge University Hospitals, NHS Trust, Cambridge CB2 0QQ, UK

\section{Introduction}

Alien limb phenomena are a heterogeneous group of behaviours in which one or more of a patient's limbs, usually an arm, behaves in a manner that appears purposeful or semipurposeful but is independent of the patient's reported intentions [1-5]. Patients' reactions to the unwanted motor behaviours are variable, including lack of awareness, surprise, frustration or denial of ownership of the limb itself [6]. Alien limb motor phenomena have been divided into two main groups [3]. The first group includes complex, unwilled motor acts, including intermanual conflict, mirror movements, interference, and the pushing aside of the directed limb by the autonomous limb. These movements have been described as often bimanual, and liable to occur within two scenarios: (a) the offending hand is involuntarily recruited to tasks which the patient intends to perform unimanually with the other hand and (b) the offending limb undertakes the incorrect act when desired to act in concert with the 
other hand. The second group of phenomena include simple, unwilled, quasi-reflex actions. These include autonomous reaching, grasping and utilisation behaviour, automatic limb withdrawal or levitation.

Despite ample clinical and basic research, the aetiology of alien limb remains poorly understood [5]. Case studies of patients with focal brain lesions have implicated frontal brain regions [7]. An anatomically derived classification of alien limb phenomena identified two separate syndromes [8]. One syndrome associated with frontal callosal damage, with or without bilateral frontal involvement, typically presents with intermanual conflict (in which involuntary non-dominant hand activity is precipitated by the internally evoked actions of the dominant hand). The other is a syndrome seen following damage to the left frontomedial and callosal regions in which the patient's dominant hand exhibits unintentional reaching and grasping evoked by external stimuli [8]. Within these frontal regions, damage to the presupplementary motor area may play a central role in the development of alien limb $[9,10]$.

Alien limb is a common clinical feature of the corticobasal syndrome. Corticobasal syndrome classically arises from the specific pathology termed 'corticobasal degeneration' [11], which is associated with aggregation of hyperphosphorylated 4-repeat tau protein [12]. Corticobasal syndrome can also arise from other neuropathologies, such as Alzheimertype pathology or progressive supranuclear palsy [13-15], Creutzfeldt-Jakob disease [14] or the cumulative effects of cerebrovascular disease [16]. The corticobasal syndrome can present with subcortical motor features (including akinetic rigidity, dystonia and myoclonus) or cortical features (including visuospatial and deficits, non-fluency and cortical sensory loss) $[17,18]$. The current international consensus clinical diagnostic criteria for corticobasal syndrome include alien limb [19], as have earlier diagnostic criteria [20, 21].

Another disorder of the sensorimotor system that is included in the diagnostic criteria of, and is common in, the corticobasal syndrome is called 'apraxia' $[7,9,10$, 22]. Apraxia may be defined as a disorder of "the execution of learned movement which cannot be accounted for either by weakness, incoordination or sensory loss, or by incomprehension of or inattention to commands" [23]. It is assessed through the pantomiming of actions (performing an action on request without example) and imitation of gestures [18]. It can result from focal lesions, classically in the corpus callosum and dominant hemisphere [23, 24], as well as neurodegenerative disorders such as corticobasal syndrome $[18,19]$. Limb apraxia is usually considered in three forms, often called 'ideational', 'ideomotor', and 'limb-kinetic apraxia' $[18,25]$. Ideational apraxia results from a conceptual deficit of the desired action. Ideomotor apraxia is a failure to convert the concept of the action into a motor programme to execute the necessary movements; it usually manifests itself through spatiotemporal errors. Limbkinetic apraxia describes reduced dexterity predominantly affecting fine movements through incoordination of the fingers, beyond that attributable to elementary motor disorders such as pyramidal dysfunction, rigidity, ataxia, tremor or dystonia [26]. Ideomotor and limb-kinetic apraxias appear to be the most common and may co-exist in the corticobasal syndrome [26], although isolated examination of apraxia in patients with co-existent rigidity, dystonia or tremor is often difficult $[18,19]$.

In addition to their co-occurrence in the corticobasal syndrome, limb apraxia and alien limb syndrome are both disorders of complex sensorimotor function with some similarities. First, shared anatomic substrates have been implicated through association with lesions either directly affecting the supplementary motor, prefrontal and parietal cortices or causing their disconnection $[7,9,10,27,28]$. Second, classical alien behaviours were phenomenologically described as 'diagonistic dyspraxia' [27] and 'magnetic apraxia' [29]. Third, in patients with corticobasal syndrome, both apraxia severity and alien limb symptoms have been associated with an implicit measure of the sense of agency [10]. However, there are clear differences between these clinical entities. Apraxia describes aberrant voluntary movement, whereas the anarchic action of an alien limb is experienced by the patient as involuntary. An alien limb is associated with a sense of foreignness and loss of agency, which is not characteristic of apraxia.

Here, we investigated the phenomenological nature of alien limb symptoms in the corticobasal syndrome and their possible link with apraxia. We performed structured examinations of praxis, motor function, cognition and alien limb in patients with corticobasal syndrome. We summarised the rate of different alien limb symptoms. We then used Bayesian analyses to compare two alternate hypotheses: (1) that alien limb phenomena and apraxia are related to each other versus (2) that alien limb phenomena are not related, in that there is no association between alien limb phenomena and the severity of apraxia. This evidence-based null result would suggest differences in terms of their functional anatomy and physiology despite co-occurrence in the corticobasal syndrome.

\section{Methods}

Participants were recruited from a specialist regional neurology clinic for cognitive and movement disorders during a 40-month period, serving a population of approximately one and a half million. Patients met the diagnosis of probable corticobasal syndrome by clinical criteria confirmed at the time of assessment rather than first presentation [17], and were later re-diagnosed under the revised consensus criteria 
[19], as probable corticobasal syndrome with either probable or definite corticobasal degeneration. Magnetic resonance imaging in each case had not indicated alternative diagnoses. The age, sex and premorbid handedness of participants are summarised in Table 1. Twenty-five patients with possible or probable progressive supranuclear palsy syndrome (PSP) were recruited as a disease control group under the former NINDS-SPSP criteria for PSP [30]. Unlike the current MDS criteria for PSP [31], the former criteria excluded overlap syndromes such as PSP-corticobasal syndrome. The research was carried out in accordance with guidelines and regulations approved by the Cambridgeshire 2 Research Ethics Committee (now 'East of England Cambridge Central'), who approved the experimental protocols. All participants gave full, informed, written consent before the experiment.

The cognitive assessment included neuropsychological evaluation with the Revised Addenbrooke's Cognitive Examination [32, 33], the Mini-Mental State Examination [34], and the Frontal Assessment Battery [35]. Motor function was evaluated using part III of the Unified Parkinson's Disease Rating Scale [36] and recording of myoclonus, mirror movements, dystonia and dyskinesia.
On our assessment of apraxia, we focused on a tailored bedside examination scored according to the broad definition of apraxia without attempting to separate subtypes because of the inherent difficulties of clinical differentiation in a complex movement disorder. Praxis was assessed using a structured bilateral five-part examination. This included the miming of three transitive and three intransitive unilateral representational actions, testing each in both the left and right hands separately (six left, six right, instructed verbally), and the imitation of six unilateral non-representational hand configurations (three left, three right, gestures demonstrated by the examiner) and the imitation of two bimanual hand configurations. Orofacial mime (to mime a kiss and yawn, instructed verbally) was also assessed. Each of the 20 actions was scored 1 in the absence of apraxic error and 0 if apraxic errors were observed. For the association with alien limb (below), we focused on limb praxis score (range: 1-18). We opted for the simplest (binary) approach, accepting it as a crude reduction of sophisticated action control without the need to arbitrarily attempt to quantify degrees of correctness or error by the examiner. This is particularly important
Table 1 Demographic, neuropsychological and behavioural data

\begin{tabular}{|c|c|c|c|c|c|c|c|c|}
\hline & \multirow[t]{2}{*}{ Units } & \multicolumn{3}{|l|}{ PSP } & \multicolumn{3}{|l|}{ CBS } & \multirow[t]{2}{*}{$p$ value ( $t$ test, $\chi^{2}$ ) } \\
\hline & & Mean/\% & SD & $N$ & Mean/\% & SD & $N$ & \\
\hline \multicolumn{9}{|l|}{ Demography } \\
\hline Age & Years & 70.6 & 7.9 & 25 & 70.3 & 9.1 & 30 & ns \\
\hline Sex & $\%$ Male & 60 & & 25 & 37 & & 30 & ns \\
\hline Handedness & $\% \mathrm{R}$ & 96 & & 25 & 97 & & 30 & ns \\
\hline \multicolumn{9}{|l|}{ Neuropsychology } \\
\hline ACE-R total & $/ 100$ & 79.1 & 10.4 & 25 & 70.0 & 13.7 & 29 & 0.008 \\
\hline Attention and orientation & $/ 18$ & 16.4 & 1.8 & 25 & 15.0 & 2.7 & 29 & 0.028 \\
\hline Memory & $/ 26$ & 21.1 & 4.3 & 25 & 17.2 & 4.7 & 29 & 0.002 \\
\hline Verbal fluency & $/ 14$ & 5.6 & 3.2 & 25 & 7.6 & 2.9 & 29 & 0.027 \\
\hline Language & $/ 26$ & 23.0 & 2.3 & 25 & 21.9 & 2.5 & 29 & ns \\
\hline Visuospatial & $/ 16$ & 13.4 & 4.3 & 25 & 8.4 & 4.5 & 29 & $<0.001$ \\
\hline MMSE & 130 & 26.4 & 2.8 & 25 & 23.1 & 4.5 & 30 & 0.002 \\
\hline FAB & $/ 18$ & 10.9 & 4.3 & 25 & 10.4 & 4.7 & 28 & ns \\
\hline \multicolumn{9}{|l|}{ Motor examination } \\
\hline UPDRS-III & $/ 108$ & 32.2 & 13.3 & 25 & 24.7 & 9.9 & 19 & 0.038 \\
\hline Praxis total limb & $/ 20$ & 19.1 & 2.1 & 24 & 9.3 & 5.2 & 30 & $<0.001$ \\
\hline Transitive mime & $/ 6$ & 5.7 & 1.0 & 24 & 3.0 & 2.0 & 30 & $<0.001$ \\
\hline Intransitive mime & 16 & 5.9 & 0.4 & 24 & 3.7 & 1.9 & 30 & $<0.001$ \\
\hline Unilateral imitation & $/ 6$ & 5.7 & 0.9 & 24 & 2.2 & 1.8 & 30 & $<0.001$ \\
\hline Bilateral imitation & 12 & 1.8 & 0.6 & 24 & 0.4 & 0.7 & 30 & $<0.001$ \\
\hline Orofacial & $/ 2$ & 2.00 & 0.0 & 22 & 1.79 & 0.6 & 28 & ns \\
\hline Alien limb questionnaire & $/ 13$ & 0.08 & 0.4 & 25 & 4.23 & 3.1 & 30 & $<0.001$ \\
\hline
\end{tabular}

$P S P$-RS Progressive supranuclear palsy Richardson syndrome, $C B S$ corticobasal syndrome, $A C E-R$ Addenbrooke's Cognitive Examination-Revised, MMSE Mini-Mental State Examination, FAB Frontal Assessment Battery, UPDRS-III Unified Parkinson's Disease Rating Scale (part III motor subscale)

$p$ values $<0.05$ were considered statistically significant 
when a complex movement disorder is present and the observer may already be attempting to discount the effect of fixed dystonia or tremor which affects the study cohort heterogeneously.

We assessed the presence of alien limb phenomena using a screening questionnaire to guide a structured history from both the patient and an accompanying relative or carer who could corroborate and prompt recall of specific events. This approach was chosen, as alien limb events are intermittent and hard to provoke (unlike apraxia), making it necessary to rely on recalled events. All of our corticobasal syndrome patients had the mental capacity to report their symptoms, and we sought to focus on the patient's own experience of agency which is crucial for characterising and diagnosing alien limb [2]. The questionnaire included a set of 13 possible experiences occurring within the past 6 months. The questions addressed different forms of alien limb phenomena: autonomous and disobedient limb movement, the interference of tasks, the sense of control and ownership and other sensations relating to the limb. The questions were: (1) Does your hand copy the other hand on its own?* (2) Does your hand ever float up in the air on its own?* (3) Do you prefer to hold your hand with the other hand? (4) Does your hand feel fidgety or restless to you? (emphasis on the feel) (5) Does your hand sometimes reach or touch things without you intending it to?* (6) Does your hand touch your face? (7) Do you prefer to keep your hand in your pocket? (8) Does your hand ever try to stop the 'good' hand doing what you want it to do?* (9) Does your hand ever reach or touch things even when you want it not to?* (10) Do you ever wish your hand would go away? (11) Does your hand sometimes feel that does not belong to you?* (12) Does your hand feel like it belongs to somebody else? (13) Does it feel as though someone else is controlling your hand? The six questions marked with an asterisk refer to phenomena which we considered most specific to alien limb, including anarchic hand phenomena, intermanual conflict and loss of the sense of ownership. We also completed the structured praxis examination and alien limb questionnaire with the PSP cohort for validation.

Logistic and linear regression analyses were used to test for an association between limb apraxia severity (measured through the structured praxis examination) and alien limb. Age and ACE-R scores were accounted for in the models as covariates. The main analyses focused on the specific alien limb symptoms reported by patients. All variables were $\mathrm{z}$-score scaled before entering in the regression analyses. Statistical analyses were performed in R [37]. Bayesian estimation of regression coefficients and calculation of Bayes factor were performed with the 'brms' package, with priors set to default weakly informative priors [38]. All plots were generated with ggplot2 package [39].

\section{Results}

Patient demographics and clinical information are summarised in Table 1. In terms of age and gender, our PSP and corticobasal syndrome patients were similar to those reported in a larger population-representative epidemiological study [40]. Limb praxis scores were incomplete for one patient with corticobasal syndrome and another with PSP. All patients completed the alien limb questionnaire.

\section{Praxis scores}

Apraxia was very common in our corticobasal syndrome cohort, with only $1 / 30$ patients (3\%) demonstrating normal praxis. In corticobasal syndrome, praxis scores ranged between $0 / 20$ (inability to perform any of the required movements) and 20/20 (no deficit) (Fig. 1a). As shown in Table 1, patients with corticobasal syndrome were significantly apraxic in all limb movements tested, resulting in mean total limb praxis score of 9.3/20 (SD 5.2). Of the 29 patients with apraxia, the left hand was preferentially affected in 23 patients (79\%), the right hand in $4(14 \%)$ and symmetrical in 2 patients (7\%). The only left-handed corticobasal syndrome patient exhibited predominantly left-sided apraxia. By contrast, most patients with PSP did not have apraxia. Seventeen of twenty-four patients (71\%) with PSP scored 20/20 (no deficit) and three more (12\%) scored 19/20.

\section{Alien limb phenomena}

Alien limb phenomena were reported in the majority of corticobasal syndrome patients (Fig. 1b) but only one person with PSP (who reported only the non-specific symptom that he had a fidgety hand that he tended to hold it). Further analyses are restricted to the corticobasal syndrome group.

Overall, 25/30 (83\%) patients with corticobasal syndrome reported at least one symptom of alien limb, and 21/30 (70\%) patients reported at least one of the more specific symptoms. These specific symptoms included motor phenomena and whether the patient feels that their limb belongs to them (see "Methods"). Each individual item received affirmative responses from some patients and their frequency is summarised in Fig. 2. Common responses were of the tendency to hold the offending hand with the better hand $(16 / 30 ; 53 \%)$, for unwilled arm levitation (14/30; 47\%), and the sensation that the limb was not theirs $(15 / 30 ; 50 \%)$. Patients rarely reported preferring to keep their bad hand restricted in a pocket $(6 / 30 ; 20 \%)$, the bad hand blocking the good hand (intermanual interference) $(5 / 30 ; 17 \%)$, the sensation that the limb belonged to somebody else $(5 / 30 ; 17 \%)$ or that the limb was under the control of another agent $(5 / 30 ; 17 \%)$. Four of 
Fig. 1 a The frequency distribution (in percentage) of apraxia score in corticobasal syndrome patients. Lower scores indicate fewer movements successfully performed (that is more abnormal behaviour). b Same as (a) but for the overall number of alien limb phenomena reported by patients (higher number indicates more alien limb symptoms reported)
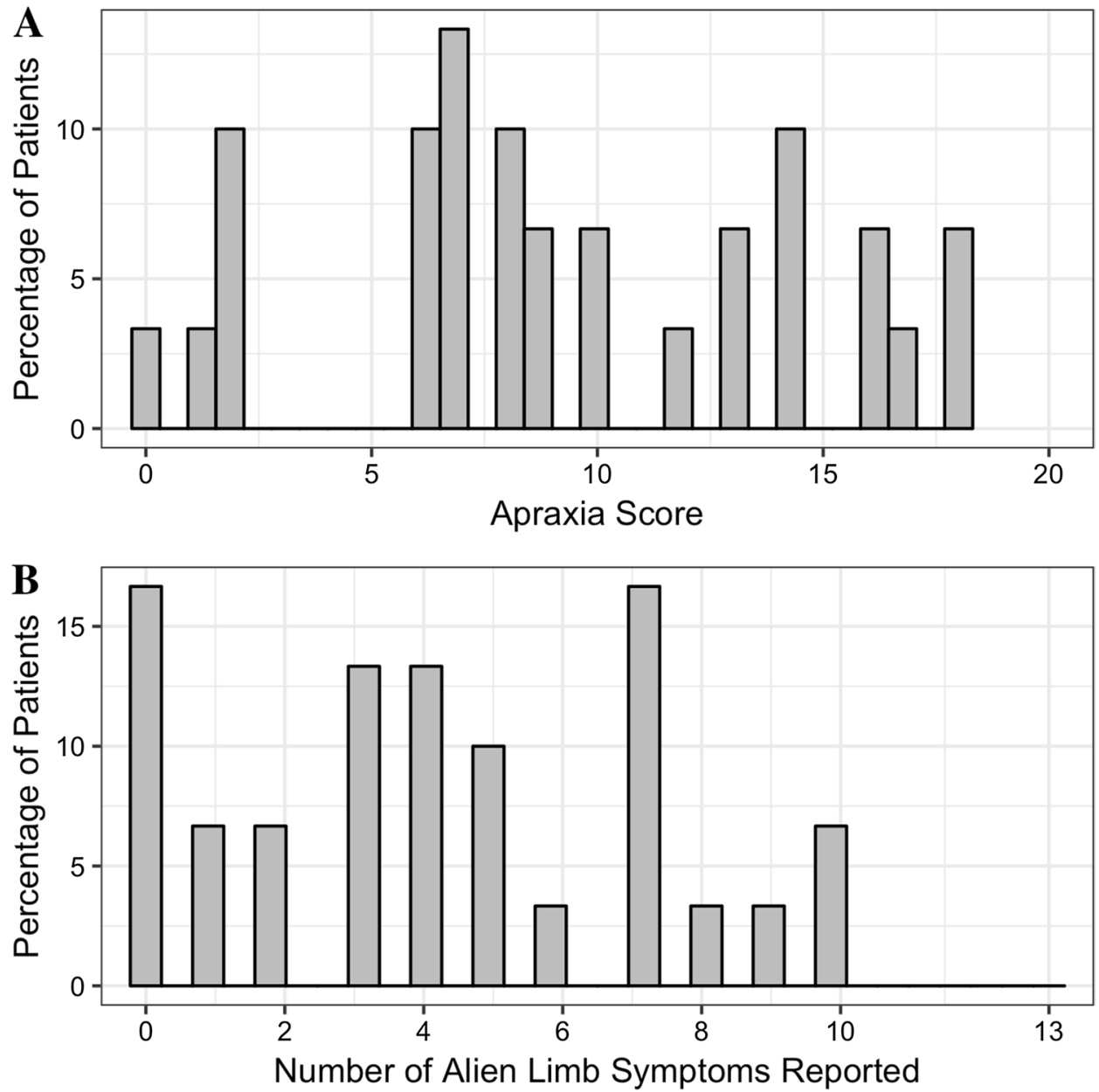

the five patients reporting either of these last two symptoms (80\%) reported both. We confirmed there was no association between cognitive impairments, measured with the ACE-R, and both the number of specific (Spearman's rho $=0.022$, $p=0.909$ ) and overall (Spearman's rho $=0.202, p=0.294$ ) alien limb phenomena reported.

\section{Association between alien limb and apraxia}

We investigated a possible association between alien limb phenomena and limb apraxia in corticobasal syndrome (Fig. 3a), while accounting for age and ACE-R score. First, we conducted a logistic regression analysis to test whether apraxia severity was associated with the occurrence of alien limb in patients. There was no association between praxis score and the emergence of at least one of the specific alien limb symptoms in patients $(z=-0.726, p=0.468$; $\mathrm{OR}=0.922,95 \% \mathrm{CI}=0.727-1.142)$. The results $\mathrm{did}$ not change when we looked at the occurrence of at least one of any of the items in the alien limb questionnaire items $(z=-0.052, p=0.958 ; \mathrm{OR}=0.992,95 \% \mathrm{CI}=0.729-1.327)$.
We performed a Bayesian estimation of the logistic regression coefficients for predicting the occurrence of at least one of the specific alien limb symptom (Fig. 3b). This analysis revealed that the probability for increased risk of developing at least one of the specific alien limb phenomena with increasing apraxia severity was 0.225 . Model comparison showed that a logistic regression model that did not include apraxia severity as an independent variable was favoured over a model that did, with a Bayesian factor of 3.68 (moderate evidence in favour of the null).

Next, we examined the phenomenological association between alien limb and apraxia by performing a linear regression analyses on praxis score predicting the number of alien limb symptoms (Fig. 3b). No such association emerged, both when looking at the number of specific alien limb phenomena reported (beta $=-0.415$, $p=0.078$ ) and the overall number of phenomena reported (beta $=-0.265, p=0.240$ ). A Bayesian estimation of the regression coefficients (Fig. 3c) showed that the probability of apraxia severity positively predicting the number of specific alien limb phenomena reported (i.e. beta $>0$ ) is 0.037. Model comparison showed that a linear regression 
Fig. 2 The frequency (in percentage) of each individual alien limb symptom in corticobasal syndrome patients. More specific alien limb symptoms are in bold-italics. Symptoms are ordered alphabetically

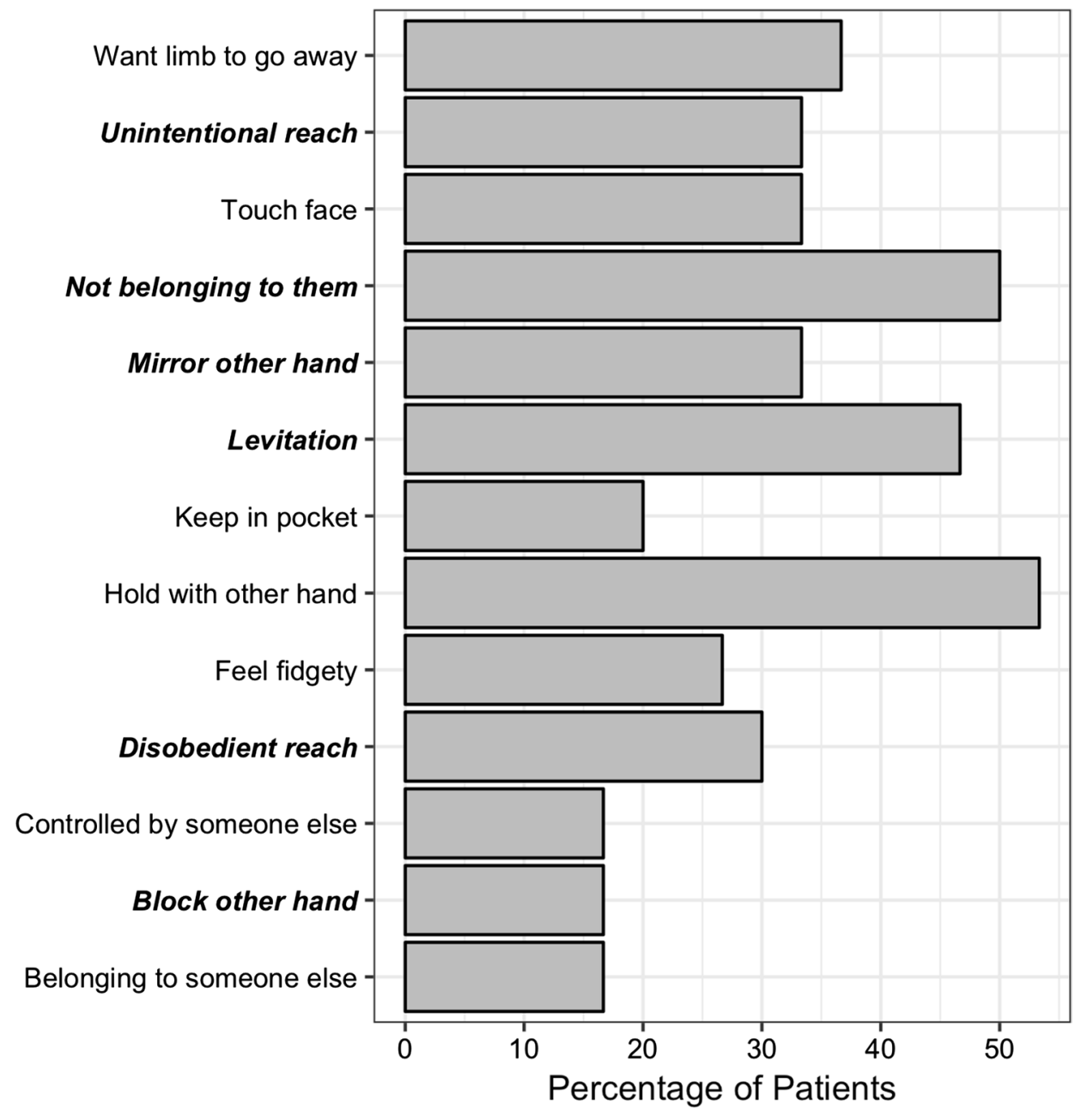

model that did not include apraxia severity as an independent variable was strongly favoured over a model that did with a Bayesian factor of 689.73 (very strong evidence, by conventional thresholds).

For completeness, we considered a non-linear relationship between alien limb and apraxia by performing non-parametric, ranked partial correlation analyses. No correlation was found between apraxia severity and the total (Spearman's rho $=-0.253, p=0.194$ ) or specific (Spearman's rho $=-0.358, p=0.061$ ) alien limb phenomena reported.

As most of the patients suffered from asymmetric apraxia, we completed the analyses with a Bayesian estimation of regression coefficients with lateralised apraxia score instead of total limb apraxia score. This revealed that the probability for a positive association between unilateral apraxia severity and occurrence of alien limb was 0.019 and 0.039 (specific and any alien limb symptom, respectively). Similarly, the probability for a positive association between unilateral apraxia severity and number of alien limb symptoms reported was 0.006 and 0.015 (specific and all alien limb symptoms, respectively).

\section{Discussion}

The principal results from this study are although both alien limb and apraxia are common in corticobasal syndrome, severe apraxia does not predict the occurrence of alien limb and there was no association between the number of alien limb phenomena reported and apraxia severity. These results have the caveat that our data emphasise the qualitative features of alien limb phenomena reported by patients, rather than their frequency or severity. We used a structured assessment of both apraxia and alien limb syndrome to formalise the assessment of these disorders for analysis and found them to be robust to the presence of another severe extrapyramidal disorder with involuntary movements: PSP. Whilst our semi-quantitative analysis did not address each form of apraxia separately, the composite limb apraxia score included imitation and mime tasks. These are sensitive to both ideomotor and limb-kinetic apraxia and also in the case of mime, ideational apraxia [18]. We first consider the implications of our results for the understanding of alien limb phenomena and apraxia, 
Fig. 3 Alien limb and apraxia. a The number of alien limb symptoms reported by patients plotted against apraxia severity scores. b Probability density function for the beta coefficient for apraxia predicting the occurrence of at least one of the specific alien limb symptoms in the logistic regression analysis, estimated with a Bayesian model fit. Vertical black line indicates the mode. Grey fill indicates $95 \%$ credible interval. c Same as (b) but for the beta coefficient of apraxia predicting the total number of specific alien limb symptoms in the linear regression analysis

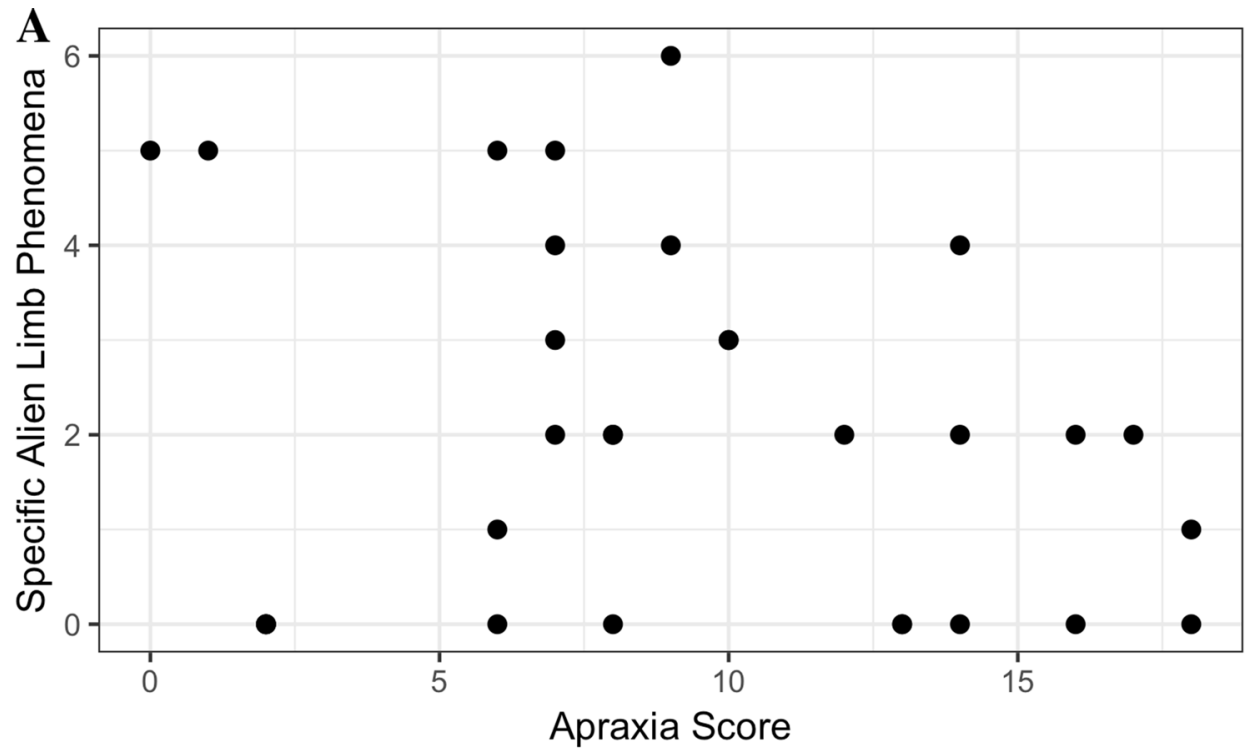

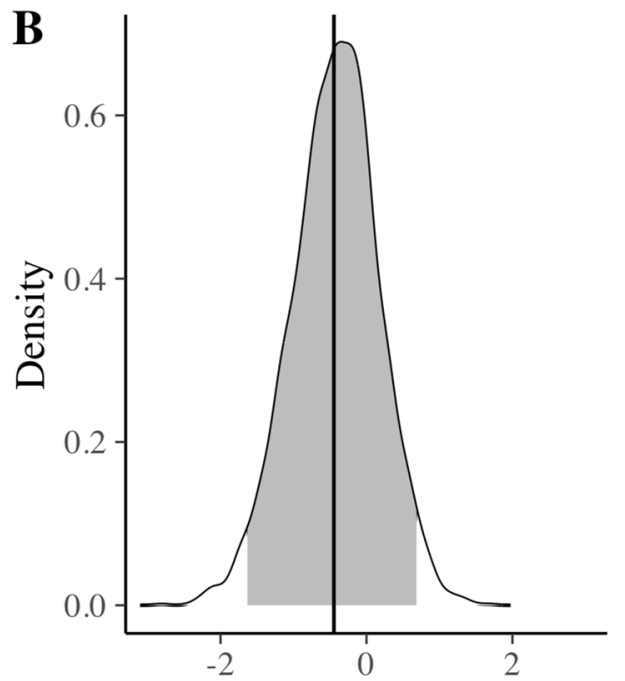

Apraxia beta logistic regression

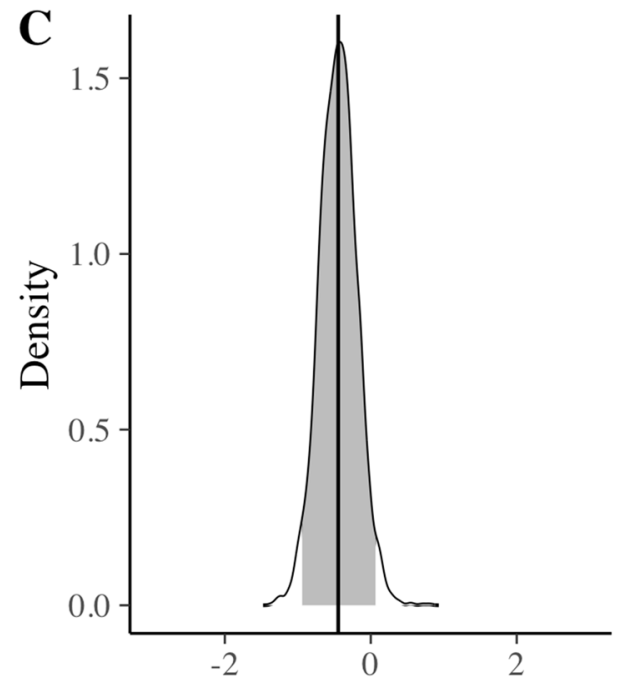

Apraxia beta linear regression before considering the relationship between these clinical entities.

\section{Alien limb phenomena}

Alien limb phenomena were common in our patients with corticobasal syndrome, with 21/30 (70\%) reporting at least one of the six specific phenomena (copying, flotation, unintentional reach, oppositional reach, interference, sense of belonging). In our patients, the actions made by alien limbs appeared semi-purposeful and not perseverative as those described due to other aetiologies [3, 6]. Moreover, none of our patients demonstrated self-destructive behaviours as those depicted in case reports arising from other aetiologies $[41,42]$, suggesting that such phenomena are uncommon in corticobasal syndrome.
The phenomenology of alien limb in our patients raises questions about body ownership deficits as in asomatognosia, body integrity dysphoria (BID) and neglect. In BID, patients feel that one or more limbs do not belong to them, together with a desire for amputation [43]. Importantly, this diminished sense of ownership is not accompanied by involuntary movements as in alien limb. Conversely, the majority of patients with alien limb in the context of corticobasal syndrome do not describe BID. While patients with corticobasal syndrome may have body part agnosia when asked to identify the area that is touched by an examiner, the errors are more of a misidentification (e.g. thumb, then ring finger touched), not a neglect or extinction of the body part as in asomatognosia. The phenomenon of neglect per se is characterised by negative, rather than positive features. Typically, it neither manifests through involuntary movements nor a 
sense of foreignness, but rather by a failure to be aware of, or attend to part of the body or external space, or by failure to use a non-paretic limb [44].

A prominent view of alien limb stresses the need for both 'foreignness' and involuntary motor activity [2]. On this view, these features are necessary to differentiate alien limb phenomena from purely motor disorders, such as dystonia, grasp reflex, athetosis, hemiballismus, hemiataxia and utilisation behaviour, as well as from sensory neglect syndromes [2]. Half of our patients met this definition of an alien hand, experiencing a loss of sense of ownership - that is, the feeling that their hand did not belong to them, together with unwanted movements (i.e. motor phenomena). By contrast, six other patients could be considered to have an 'anarchic hand syndrome', as they reported specific motor phenomena while maintaining a sense of ownership [45]. Each of the 15 patients who reported the sensation that their limb did not belong to them also reported at least one specific motor phenomenon. This finding suggests that unwilled motor behaviour may be necessary, but not sufficient for the loss of ownership.

Longitudinal data will be required to investigate whether alien limb results from a two-step process, where a patient first develops an anarchic limb and subsequently loses their sense of ownership. Importantly, while half of our patients reported a feeling of loss of limb ownership, only a third of these reported a sensation of possession or passivity. Therefore, the type of perceived loss of control observed in our patients is distinct from the classic somatic delusions of control which may be experienced by patients with psychosis, and some reported patients with alien limb syndromes of other etiologies [2, 6, 8, 41, 42].

More than a third of our patients reported unintentional reaching movements. Reaching and grasping movements of an alien limb have been suggested to result from 'exaggerated affordance', in which motor schema are abnormally disinhibited [46]. These motor plans are considered automatic as they are stimulus (e.g. object) driven, and in the case of alien limb, a failure to inhibit such motor plans leads to unwanted movements [46].

\section{Apraxia}

Nearly all patients with corticobasal syndrome were found to be apraxic before the degree of akinesia, rigidity, dystonia or myoclonus precluded interpretation of motor deficits as apraxic. 26/29 of the affected patients (90\%) had significant asymmetry in their apraxia. This is consistent with the common presenting complaint (in corticobasal syndrome) of unilateral 'clumsiness' of hand movements. The impact on mime, imitation and tool use was not simply a function of the complexity of the desired action, suggesting the observed motor deficits in corticobasal syndrome are likely to be attributable to limb-kinetic apraxia $[18,25]$. Our series also found left-sided predominance of asymmetrical apraxia. This is reminiscent of the apraxia from callosal lesions, which has been attributed to interhemispheric disconnection affecting the transfer of motor programmes from the dominant to non-dominant hemisphere [23].

With our test battery for apraxia, we sought to encompass a range of simple tasks, of mime and copy, transitive and intransitive, and to do so in a timeframe that was not fatiguing or prone to missing data. The battery was quick, but covered the principal types of dyspraxic deficit. Given the high prevalence and clear severity of apraxia in corticobasal syndrome, the current range of tests was sufficient to reveal a wide range of type and severity of apraxia. This variance is of course key to the power to detect covariance with alien limb in our study. People with PSP in our study, on the other hand, had more severe UPDRS-III scores than our patients with corticobasal syndrome (see Table 1), yet they performed our apraxia battery well, suggesting that the battery was not simply identifying motor signs of parkinsonian movement disorders.

Our apraxia test battery did not attempt to make inferences of ideational, ideomotor and limb-kinetic subtypes. There are several reasons for this. First, we did not assume that the presentations of apraxia would be the same in a chronic degenerative and distributed disease as in acute, focal surgical or stroke lesions. The complexity of motor deficits arising from combined cortical and basal ganglia degeneration might obscure the classical dissociations and interpretation of apraxic movements [18, 25]. It has been suggested that ideomotor apraxia is the most common apraxia in corticobasal syndrome [19], while ideational apraxia (revealed by identification and recognition) is less common in corticobasal syndrome [18]. It remains uncertain to what extent the poverty of fine finger movements in corticobasal syndrome is attributable to limb-kinetic apraxia as a true form of apraxia, as opposed to other concurrent pyramidal and extrapyramidal impairments [18, 25]. Moreover, limb-kinetic apraxia in corticobasal syndrome often coexists with ideomotor apraxia [26]. Hence, we opted for a functional description in terms of the tasks and an aggregate score of the deficits rather than subtyping the apraxia in corticobasal syndrome.

\section{The relationship between alien limb phenomena and apraxia}

Alien limb and apraxia have been both associated with changes in implicit measures of awareness and control of action [10]. Their co-occurrence and shared anatomical substrates suggested that they might be mechanistically related. However, in our cross-sectional cohort of patients with corticobasal syndrome, we found no association between the 
occurrence or the number of reported alien limb phenomena and limb apraxia severity. Bayesian analyses and model comparison showed that apraxia severity is very unlikely to be a positive predictor of alien limb symptoms. These results do not support the hypothesis that severe apraxia leads to alien limb. Instead, our findings on the phenomenology of alien limb are consistent with a syndrome in which a patient first presents with an involuntary motor disorder and subsequently develops a sensation of 'foreignness' or 'alienness'. The emergence of the motor phenomena of alien limb, even those which have previously been considered dyspraxic in nature [27, 29], appears to bear no simple association with the current severity of apraxia.

The clinical and phenomenological dissociation between apraxia and alien limb phenomena might be surprising, as they both share underlying brain lesions, e.g. in the supplementary motor, prefrontal and parietal cortices or white matter connecting these regions $[7,9,10,27,28]$. What might explain this clinical dissociation, considering, despite the shared anatomical lesions? First, alien limb and apraxia may result from overlapping, but not identical underlying brain lesions [7, 22]. Specifically, the pre-supplementary motor area plays a key role within a prefrontal network critical to both alien limb and apraxia. However, the exact localisation of brain lesions within this network may determine the specific manifestation of alien limb, apraxia or both [10]. A disconnection of this prefrontal network from posterior parietal regions that integrate spatiotemporal signals may lead to apraxia [10,47], whereas damage to internal feedback loops may lead to an inappropriate activation of motor schema that result in alien limb phenomena [10,46, 48].

Second, different pathological processes may selectively affect the functions of a shared network mediating praxis and voluntary movement. Our patients had clinically defined corticobasal syndrome, with neurodegeneration (rather than metabolic or cerebrovascular disease), but pathological heterogeneity is common within this syndrome $[13,15]$. It is conceivable that different pathological mechanisms could cause corticobasal syndromes with different motor disorders, although the presence of corticobasal degeneration vs Alzheimer pathology is not directly distinguished by alien limb or apraxia $[13,15]$.

A third possibility is a complex temporal relationship during disease progression in which apraxia and alien limb phenomena develop out of phase. The relationship between apraxia and alien limb phenomena may then not be evident in a cross-sectional study. For example, as praxis worsens, alien limb phenomena might diminish due to their obscuration by dystonia or akinetic rigidity [17]. In the three patients with serial praxis examinations and alien limb questionnaires, praxis always deteriorated while alien limb phenomena emerged and sometimes disappeared again over time (data not shown). One might speculate that as the pathways which mediate praxis degenerate in corticobasal syndrome, transient imbalances occur between networks for motor control, attention and awareness which lead to manifestations of alien limb phenomena. The independence of corticobasal alien limb phenomena from apraxia may therefore not simply generalise to cases with other aetiologies, such as brain lesions that concurrently affect regions common to both disorders.

Our study has several limitations. First, unlike the examination of apraxia, our assessment of alien limb symptoms relied on a structured questionnaire. This is because alien limb symptoms are intermittent and hard to provoke in the clinic (unlike apraxia). Responses were corroborated by the carer, and we assessed PSP as a control patient group, which as expected reported no alien limb symptoms. Within the corticobasal syndrome group, certain responses were consistently rare, such as sensation of possession, which could serve as negative controls. Second, we quantified the number of distinct alien limb features, not their frequency or functional impact. In pilot work, patients and their companions found reporting of the frequency of paroxysmal alien phenomena difficult. This may reflect lack of awareness of some episodes, recall bias for episodes that were socially distressing, or the influence of other cognitive and motor features on the functional impact of alien limb. Moreover, we prioritised the description of actual alien limb phenomena, which are not operationalised in the current consensus diagnostic criteria [19]. Importantly, this would not affect our investigation into the link between apraxia severity and the occurrence (rather than severity) of alien limb. Third, our cross-sectional study is unable to assess the timeline for the development of apraxia and alien limb, for which longitudinal studies are needed.

\section{Conclusions}

Alien limb phenomena and apraxia are both common disorders in the corticobasal syndrome. We describe the phenomenology of alien limb in the corticobasal syndrome, as reported by patients and apraxia as observed by clinicians. Our data do not support the interpretation of alien limb phenomena resulting from apraxia in neurodegenerative disease. There might be qualitative differences from the alien limb phenomena and apraxia following focal brain lesions, but such features are less well characterised than in corticobasal syndrome where alien limb and apraxia are both diagnostic features. Longitudinal and quantitative studies are needed to confirm that the alien limb is a transient feature, whereas apraxia declines continuously, independent from one another. 
Acknowledgements The work was supported by the Guarantors of Brain Entry Fellowship (DJLS), the James S. McDonnell Foundation 21st Century Science Initiative, Scholar Award in Understanding Human Cognition (JBR), the Wellcome Trust [103838] and the Medical Research Council [SUAG/004 RG91365 JBR, and G0700503 BCPG], the National Institute for Health Research Cambridge Biomedical Research Centre including the Cambridge Brain Bank, the Raymond and Beverley Sackler Trust, and the Cambridge Centre for Parkinson-plus.

\section{Compliance with ethical standards}

Conflicts of interest JBR serves as an associate editor to Brain, and is a non-remunerated trustee of the Guarantors of Brain and the PSP Association (UK). He provides consultancy to Asceneuron, Biogen, UCB and has research grants from AZ-Medimmune, Janssen, Lilly as industry partners in the Dementias Platform UK.

Ethical approval The research was carried out in accordance with guidelines and regulations approved by the Cambridgeshire 2 Research Ethics Committee (now 'East of England-Cambridge Central'), who approved the experimental protocols. All participants gave full, informed, written consent before the experiment.

Open Access This article is licensed under a Creative Commons Attribution 4.0 International License, which permits use, sharing, adaptation, distribution and reproduction in any medium or format, as long as you give appropriate credit to the original author(s) and the source, provide a link to the Creative Commons licence, and indicate if changes were made. The images or other third party material in this article are included in the article's Creative Commons licence, unless indicated otherwise in a credit line to the material. If material is not included in the article's Creative Commons licence and your intended use is not permitted by statutory regulation or exceeds the permitted use, you will need to obtain permission directly from the copyright holder. To view a copy of this licence, visit http://creativecommons.org/licenses/by/4.0/.

\section{References}

1. Biran I, Giovannetti T, Buxbaum L, Chatterjee A (2006) The alien hand syndrome: what makes the alien hand alien? Cogn Neuropsychol 23:563-582. https://doi.org/10.1080/0264329050 0180282

2. Doody RS, Jankovic J (1992) The alien hand and related signs. J Neurol Neurosurg Psychiatry 55:806-810. https://doi. org/10.1136/jnnp.55.9.806

3. Fisher CM (2000) Alien hand phenomena: a review with the addition of six personal cases. Can J Neurol Sci J Can Sci Neurol 27:192-203. https://doi.org/10.1017/S0317167100000834

4. Josephs KA, Rossor MN (2004) The alien limb. Pract Neurol 4:44-45. https://doi.org/10.1111/j.1474-7766.2004.06-189.x

5. Rowe JB, Wolpe N (2014) Disorders of volition from neurological disease: altered awareness of action in neurological disorders. In: Eitam B, Haggard P (eds) The sense of agency. Oxford University Press, Oxford

6. Biran I, Chatterjee A (2004) Alien hand syndrome. Arch Neurol 61:292-294. https://doi.org/10.1001/archneur.61.2.292

7. Scepkowski LA, Cronin-Golomb A (2003) The alien hand: cases, categorizations, and anatomical correlates. Behav Cogn Neurosci Rev 2:261-277. https://doi.org/10.1177/1534582303 260119
8. Feinberg TE, Schindler RJ, Flanagan NG, Haber LD (1992) Two alien hand syndromes. Neurology 42:19-24

9. Albrecht F, Mueller K, Ballarini T et al (2019) Unraveling corticobasal syndrome and alien limb syndrome with structural brain imaging. Cortex 117:33-40. https://doi.org/10.1016/j.corte x.2019.02.015

10. Wolpe N, Moore JW, Rae CL et al (2014) The medial frontal-prefrontal network for altered awareness and control of action in corticobasal syndrome. Brain 137:208-220. https://doi.org/10.1093/ brain/awt302

11. Rebeiz JJ, Kolodny EH, Richardson EPJ (1968) Corticodentatonigral degeneration with neuronal achromasia. Arch Neurol 18:20-33

12. Kouri N, Whitwell JL, Josephs KA et al (2011) Corticobasal degeneration: a pathologically distinct $4 \mathrm{R}$ tauopathy. Nat Rev Neurol 7:263-272. https://doi.org/10.1038/nrneurol.2011.43

13. Alexander SK, Rittman T, Xuereb JH et al (2014) Validation of the new consensus criteria for the diagnosis of corticobasal degeneration. J Neurol Neurosurg Psychiatry 85:925-929. https://doi. org/10.1136/jnnp-2013-307035

14. Boeve BF, Maraganore DM, Parisi JE et al (1999) Pathologic heterogeneity in clinically diagnosed corticobasal degeneration. Neurology 53:795-795. https://doi.org/10.1212/WNL.53.4.795

15. Shelley BP, Hodges JR, Kipps CM et al (2009) Is the pathology of corticobasal syndrome predictable in life? Mov Disord 24:1593-1599. https://doi.org/10.1002/mds.22558

16. Kreisler A, Mastain B, Tison F et al (2007) Infarctus cérébraux multiples se présentant comme une dégénérescence corticobasale : pseudo dégénérescence cortico-basale vasculaire ? Rev Neurol (Paris) 163:1191-1199. https://doi.org/10.1016/S0035 -3787(07)78403-5

17. Boeve BF, Lang AE, Litvan I (2003) Corticobasal degeneration and its relationship to progressive supranuclear palsy and frontotemporal dementia. Ann Neurol 54:S15-S19. https://doi. org/10.1002/ana.10570

18. Stamenova V, Roy EA, Black SE (2009) A model-based approach to understanding apraxia in corticobasal syndrome. Neuropsychol Rev 19:47-63. https://doi.org/10.1007/s11065-008-9079-5

19. Armstrong MJ, Litvan I, Lang AE et al (2013) Criteria for the diagnosis of corticobasal degeneration. Neurology 80:496-503. https://doi.org/10.1212/WNL.0b013e31827f0fd1

20. Kumar R, Bergeron C, Pollanen M (1998) Cortico-basal ganglionic degeneration. In: Jankovic J, Tolosa E (eds) Parkinson's disease and movement disorders. Lippincott Williams and Wilkins, Baltimore, pp 297-316

21. Litvan I, Bhatia KP, Burn DJ et al (2003) SIC Task Force appraisal of clinical diagnostic criteria for parkinsonian disorders. Mov Disord 18:467-486. https://doi.org/10.1002/mds.10459

22. Wheaton LA, Hallett M (2007) Ideomotor apraxia: a review. J Neurol Sci 260:1-10. https://doi.org/10.1016/j.jns.2007.04.014

23. Geschwind N (1975) The apraxias: neural mechanisms of disorders of learned movement. Am Sci 63:188-195

24. Liepmann H (1900) Das Krankheitshildder der Apraxie (Motorischen/Asymbolie). Monatschrift Psychiatr Neurol 8(15-44):102-132

25. Pramstaller PP, Marsden CD (1996) The basal ganglia and apraxia. Brain 119:319-340. https://doi.org/10.1093/brain/119.1.319

26. Leiguarda RC, Merello M, Nouzeilles MI et al (2003) Limbkinetic apraxia in corticobasal degeneration: clinical and kinematic features: Limb-Kinetic Apraxia. Mov Disord 18:49-59. https://doi.org/10.1002/mds.10303

27. Akelaitis AJ (1945) Studies on the corpus callosum: IV. Diagonistic dyspraxia in epileptics following partial and complete section of the corpus callosum. Am J Psychiatry 101:594-599. https://doi. org/10.1176/ajp.101.5.594 
28. Haaland KY, Harrington DL, Knight RT (2000) Neural representations of skilled movement. Brain 123:2306-2313. https://doi. org/10.1093/brain/123.11.2306

29. Denny-Brown D (1958) The nature of apraxia. J Nerv Ment Dis 126:9-32. https://doi.org/10.1097/00005053-195801000-00003

30. Litvan I, Agid Y, Calne D et al (1996) Clinical research criteria for the diagnosis of progressive supranuclear palsy (Steele-Richardson-Olszewski syndrome): report of the NINDS-SPSP international workshop. Neurology 47:1-9. https://doi.org/10.1212/ WNL.47.1.1

31. Höglinger GU, Respondek G, Stamelou M et al (2017) Clinical diagnosis of progressive supranuclear palsy: the movement disorder society criteria: MDS clinical diagnostic criteria for PSP. Mov Disord 32:853-864. https://doi.org/10.1002/mds.26987

32. Bak TH (2005) Cognitive bedside assessment in atypical parkinsonian syndromes. J Neurol Neurosurg Psychiatry 76:420-422. https://doi.org/10.1136/jnnp.2003.029595

33. Mioshi E, Dawson K, Mitchell J et al (2006) The Addenbrooke's Cognitive Examination Revised (ACE-R): a brief cognitive test battery for dementia screening. Int J Geriatr Psychiatry 21:1078 1085. https://doi.org/10.1002/gps.1610

34. Folstein MF, Folstein SE, McHugh PR (1975) "Mini-mental state". A practical method for grading the cognitive state of patients for the clinician. J Psychiatr Res 12:189-198

35. Dubois B, Slachevsky A, Litvan I, Pillon B (2000) The FAB: a frontal assessment battery at bedside. Neurology 55:1621-1626. https://doi.org/10.1212/WNL.55.11.1621

36. Fahn S, Elton R (1987) UPDRS program members. Unified Parkinsons Disease Rating Scale. In: Fahn S, Marsden C, Goldstein M, Calne D (eds) Recent developments in Parkinsons disease. Macmillan Healthcare Information, Florham Park, pp 153-163

37. R Core Team (2016) R: A language and environment for statistical computing. R Foundation for Statistical Computing, Vienna

38. Bürkner PC (2017) brms: an R package for bayesian multilevel models using stan. J Stat Softw 80:1-28. https://doi.org/10.18637 /jss.v080.i01
39. Wickham H (2009) ggplot2: elegant graphics for data analysis. Springer-Verlag, New York

40. Coyle-Gilchrist ITS, Dick KM, Patterson K et al (2016) Prevalence, characteristics, and survival of frontotemporal lobar degeneration syndromes. Neurology 86:1736-1743. https://doi. org/10.1212/WNL.0000000000002638

41. Ay H, Buonanno FS, Price BH et al (1998) Sensory alien hand syndrome: case report and review of the literature. J Neurol Neurosurg Psychiatry 65:366-369. https://doi.org/10.1136/ jnnp.65.3.366

42. Leiguarda R, Starkstein S, Nogues M et al (1993) Paroxysmal alien hand syndrome. J Neurol Neurosurg Psychiatry 56:788-792. https://doi.org/10.1136/jnnp.56.7.788

43. Brugger P, Lenggenhager B, Giummarra MJ (2013) Xenomelia: a social neuroscience view of altered bodily self-consciousness. Front Psychol. https://doi.org/10.3389/fpsyg.2013.00204

44. Rode G, Pagliari C, Huchon L et al (2017) Semiology of neglect: an update. Ann Phys Rehabil Med 60:177-185. https://doi. org/10.1016/j.rehab.2016.03.003

45. Sala CM (1998) Disentangling the Alien and Anarchic Hand. Cognit Neuropsychiatry 3:191-207

46. McBride J, Sumner P, Jackson SR et al (2013) Exaggerated object affordance and absent automatic inhibition in alien hand syndrome. Cortex. https://doi.org/10.1016/j.cortex.2013.01.004

47. Borroni B, Garibotto V, Agosti C et al (2008) White matter changes in corticobasal degeneration syndrome and correlation with limb apraxia. Arch Neurol. https://doi.org/10.1001/archn eur.65.6.796

48. Schaefer M, Heinze H-J, Galazky I (2010) Alien hand syndrome: neural correlates of movements without conscious will. PLoS ONE 5:e15010. https://doi.org/10.1371/journal.pone.0015010 